\title{
Patterns of balance loss with systematic perturbations in Parkinson's disease and multiple sclerosis
}

\author{
Diane D. Allen ${ }^{\mathrm{a}}$, Jessica Gadayan ${ }^{\mathrm{b}}$, Rebecca Hughes ${ }^{\mathrm{c}}$, Christine Magdalin ${ }^{\mathrm{d}}$, Catherine Jang ${ }^{\mathrm{e}}$, Amy \\ Schultz $^{\mathrm{f}}$, Kathryn Scott ${ }^{\mathrm{g}}$, Leah Vivero ${ }^{\mathrm{h}}$, Rolando L. Lazaro ${ }^{\mathrm{i}}$ and Gail L. Widener ${ }^{\mathrm{j}, *}$ \\ ${ }^{a}$ Graduate Program in Physical Therapy, University of California San Francisco/San Francisco State University, \\ San Francisco, CA, USA \\ ${ }^{\mathrm{b}}$ Albany Physical Therapy, Albany, CA, USA \\ ${ }^{\mathrm{c}}$ Baylor University Medical Center, Dallas, TX, USA \\ ${ }^{\mathrm{d}}$ Kaiser San Francisco, San Francisco, CA, USA \\ ${ }^{\mathrm{e}}$ Duke Health, Hanover, MD, USA \\ ${ }^{\mathrm{f}}$ Red Bull Athlete Performance Center, Red Bull Media House, Santa Monica, MA, USA \\ ${ }^{\mathrm{g}}$ Veterans Affairs Rocky Mountain Regional Health Care System, Aurora, CO, USA \\ ${ }^{\mathrm{h}}$ Department of Veterans Affairs, Milpitas, CA, USA \\ ${ }^{\mathrm{i}}$ Sacramento State University, Sacramento, CA, USA \\ j Physical Therapy, Samuel Merritt University, Oakland, CA, USA
}

Received 2 July 2021

Accepted 18 October 2021

\begin{abstract}
.
BACKGROUND: Multiple sclerosis (MS) and Parkinson's disease (PD) may affect balance differently. However, no studies have compared loss of balance (LOB) patterns following multi-directional perturbations.

OBJECTIVE: 1) determine reliability of LOB ratings following standardized manual perturbations; 2) compare LOB ratings in MS, PD, and healthy control (HC) groups following perturbations at upper/lower torso, in anterior/posterior, right/left, and rotational directions.

METHODS: 1) reviewers rated videotaped LOB following perturbations applied by 4 clinicians in 6-10 HCs. 2) three groups (64 MS, $42 \mathrm{PD}$ and $32 \mathrm{HC}$ ) received perturbations. LOB ratings following perturbations were analyzed using two-factor mixed ANOVAs for magnitude and prevalence.

RESULTS: 1) LOB ratings showed moderate to good ICC and good to excellent agreement. 2) MS group showed greater magnitude and prevalence of LOB than PD or HC groups $(p<.001)$. All groups showed greater LOB from right/left versus anterior/posterior perturbations $(p<.01)$. PD showed greater LOB from perturbations at upper versus lower torso; MS and HC showed greater LOB from posterior versus anterior perturbations.

CONCLUSIONS: Our reliable rating scale showed differences in patterns of LOB following manual perturbations in MS, PD, and HC. Clinically accessible and reliable assessment of LOB could facilitate targeted perturbation-based interventions and reduce falls in vulnerable populations.
\end{abstract}

Keywords: Parkinson's disease, multiple sclerosis, balance, perturbations, assessment, reliability

\footnotetext{
*Address for correspondence: Gail L. Widener, PT, PhD, Professor, Physical Therapy, Samuel Merritt University, Oakland, CA,
} 


\section{Introduction}

Parkinson's disease (PD) and multiple sclerosis (MS) can impair balance and increase risk of falling (Cameron \& Lord, 2010; Kalilani, Asgharnejad, Palokangas, \& Durgin, 2016; Nilsagard et al., 2015; Pickering et al., 2007). However, pathology-specific balance dysfunction likely results in variations in direction, type, and magnitude of loss of balance (LOB) following different environmental challenges. Although prior research has compared balance in PD and MS (Cattaneo et al., 2016), patterns of LOB resulting from multi-directional perturbations have not previously been examined. Assessing differences in LOB in reaction to everyday types of perturbations could help target intervention and prevent falls (Cameron \& Lord, 2010).

One easy-to-administer clinical test of reactive balance is the sternal shove test or nudge test, performed alone (Granacher, Muehlbauer, \& Gruber, 2012) or as an item in other tests (Tinetti, 1986). Subjects stand with their feet close together and attempt to maintain stable balance when the examiner pushes them on the sternum with light pressure. The examiner rates the response: $0=$ starts to fall; $1=$ feet start to move; $2=$ stance remains stable (Granacher, et al., 2012). Such a test has face validity because it mimics a bump that a patient might receive while standing in line or waiting for something, but examiners test only a posteriorly directed bump at shoulder height. Reliability has not been tested with regard to the intensity of the perturbation impulse or rating of the response (Granacher, et al., 2012).

A standardized system was developed to apply manual perturbations, similar to the nudge test, in various directions at shoulder and hip height and observe patient response. This system is described in relation to Balance-Based Torso-Weighting (BBTW) (Gibson-Horn, 2008), a therapeutic modality that has improved balance or gait in people with MS (Crittendon, O'Neill, Widener, \& Allen, 2014; Gorgas, Widener, Gibson-Horn, \& Allen, 2015; Horn, Allen, Gibson-Horn, \& Widener, 2018; Widener, Allen, \& Gibson-Horn, 2009a, 2009b), cerebellar ataxia (Widener et al., 2020), PD (Lazaro, 2010), and healthy elderly (Vincenzo, Gibson-Horn, \& Gray, 2017). For BBTW, perturbations are applied manually as nudges and rotational forces in six prescribed directions at both shoulders and pelvis. The examiner observes the direction and rates the amount of balance loss and recovery with each perturbation on a $0-3$ scale, and then strategically applies small weights (totaling less than $2 \%$ body weight) on a vest-like garment to counter the direction(s) of greatest balance loss. The BBTW set of perturbations has face validity with regard to everyday bumps compared to the nudge test, because forces are applied from different directions at shoulder and hip height. These systematic perturbations also have consequential validity because subject responses drive therapeutic application of the BBTW weights, with consequential improvements in performance of gait and balance (Horn, et al., 2018; Widener, et al., 2009b). Intrarater reliability of the intensity of perturbations has been confirmed with a dynamometer, with no significant difference in force, time to peak force, or total time of the perturbations across subjects prior to weighting with BBTW (Crittendon, et al., 2014). Inter-rater reliability has not yet been examined regarding the rating of observed LOB following these perturbations.

Balance responses following sensory challenges in PD and MS manifest differences in their pathology (Cattaneo, et al., 2016). PD leads to abnormal dopaminergic striatal projections that can increase axial rigidity and impair postural synergies; people with PD show increased postural sway when standing on foam, suggesting a reliance on proprioceptive input. In contrast, MS leads to slowed somatosensory conduction and impaired central integration of input; people with MS show excessive sway in foam and eyes-closed conditions, suggesting a reliance on visual input especially during proprioceptive challenges (Cattaneo, et al., 2016). Perturbations via support surface displacement in each diagnostic group have revealed postural vulnerabilities compared to healthy controls (Carpenter, Allum, Honegger, Adkin, \& Bloem, 2004; Dietz, Berger, \& Horstmann, 1988; Peterson, Huisinga, Spain, \& Horak, 2016), but no pathology-specific comparisons have been made for LOB following nudges that mimic everyday types of bumps and pushes.

The purpose of this descriptive study was to: a) examine the inter-rater reliability of rating LOB resulting from a standardized set of manual perturbations in different directions at the upper or lower torso of standing participants, and b) compare patterns of LOB in people with PD or MS and healthy controls (HCs) following these perturbations. The hypotheses were that acceptable reliability would be noted and that LOB patterns would differ between groups and among different directions and locations of perturbations. 


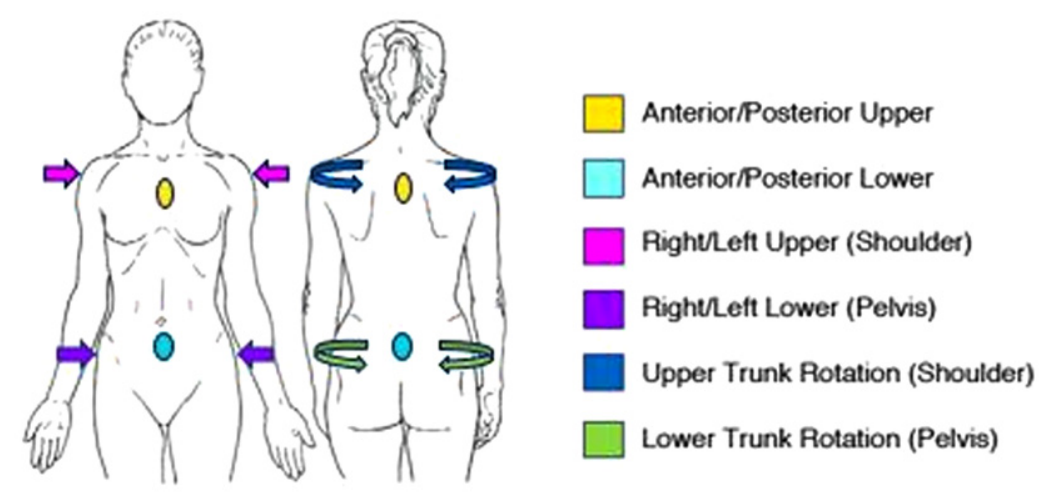

Loss of Balance Rating Scale

\begin{tabular}{|c|c|c|}
\hline Score & Anterior/Posterior/Lateral Nudges & Rotational Perturbations \\
\hline 0 & Normal; Fast & Barely moves \\
\hline 1 & Small delay; Torso moves a little & $\begin{array}{c}\text { Small movement of shoulder or } \\
\text { pelvis; No foot rotation }\end{array}$ \\
\hline 2 & $\begin{array}{c}\text { Ripple effect; Torso moves } \\
\text { moderately }\end{array}$ & $\begin{array}{c}\text { Trunk rotation }>20 \text { to }<60 \text { degrees; } \\
\text { Foot rotation }<45 \text { degrees }\end{array}$ \\
\hline 3 & $\begin{array}{c}\text { Bilateral toes or heels come up } \\
\text { significantly; Torso moves wildly; } \\
\text { Takes protective step; falls if not } \\
\text { caught }\end{array}$ & $\begin{array}{c}\text { Trunk rotation }>60 \text { degrees; } \\
\text { Foot rotation equal } 45 \text { to } 90 \\
\text { degrees or more }\end{array}$ \\
\hline
\end{tabular}

Fig. 1. Loss of balance assessment and rating scale. Locations and directions of the standardized set of perturbations includes nudges and resisted rotations.

\section{Methods}

All data were collected according to protocols approved by Samuel Merritt University and San Francisco State University, Institutional Review Boards. Written informed consent was obtained from all participants prior to study enrollment.

\subsection{Reliability}

Inter-rater reliability was assessed to determine agreement on the magnitude of balance loss following a standard set of manual perturbations. Raters viewed videotapes of subject responses to perturbations given by physical therapists.

Four physical therapists systematically applied manual perturbations and rated LOB as part of a BBTW assessment and intervention protocol. The therapists had 24-32 years of clinical experience, and 2-10 years of experience using the BBTW assessment and intervention protocol.(Crittendon, et al., 2014) The therapists applied the perturbations and rated LOB in a convenience sample of 10 healthy volunteer subjects recruited by word of mouth (aged
23-65 years; 3 male). Each subject was assessed by at least two therapists. Each therapist assessed at least six subjects. Each subject had at least 30 minutes between assessment sessions; all assessment sessions were videotaped and completed within a four-hour period.

The perturbations consisted of a standard set of nudges and resisted rotations (Fig. 1) applied while subjects stood with feet together, arms at their sides. Subjects were instructed to hold steady, "Don't let me move you." Thus, in contrast to push and release tests (Shirley Ryan AbilityLab, 2012) that specifically rate stepping responses, none of the perturbations typically induced compensatory stepping (or falls) in healthy controls. Therapists manually applied quick nudges to the anterior, posterior, left, and right, at the level of the shoulders and pelvis; thus, they applied eight nudges for each subject, with several seconds allowed for balance stabilization before each nudge. Therapists were trained to apply nudges of approximately $2-3 \mathrm{~kg}$ force, established in a previous study using a dynamometer (Crittendon, et al., 2014). Subjects were told that they would receive nudges, although the timing, location, and direction were not always directly anticipated. In addition to nudges, 
Table 1

Set-up for Studies of Reliability (a) and Comparison of Patterns of Balance Loss (b)

\begin{tabular}{|c|c|c|c|c|c|c|c|c|}
\hline \multicolumn{9}{|c|}{ a. Number of Subjects and Reviewers for Each of Four Physical Therapists (A-D) } \\
\hline \multicolumn{5}{|l|}{ Therapist } & A & $\mathrm{B}$ & $\mathrm{C}$ & $\overline{\mathrm{D}}$ \\
\hline \multicolumn{5}{|c|}{ Years of Experience with Perturbation Method } & 10 & 2 & 2 & $\overline{5}$ \\
\hline \multicolumn{5}{|l|}{ Subjects Assessed } & 10 & 7 & 7 & 6 \\
\hline \multicolumn{5}{|l|}{ Video Reviewers } & 4 & 5 & 5 & 5 \\
\hline \multicolumn{5}{|c|}{ Perturbations Assessed } & 120 & 80 & 84 & 72 \\
\hline \multicolumn{9}{|c|}{ b. Demographics for Each Group } \\
\hline Group & Male & Female & $\begin{array}{c}\text { Age } \\
\text { Mean (SD) }\end{array}$ & $\begin{array}{l}\text { Years with } \\
\text { Diagnosis } \\
\text { Mean (SD) }\end{array}$ & $\begin{array}{l}\text { Disability } \\
\text { Level } \\
\text { Mean (SD) }\end{array}$ & $\begin{array}{r}\text { Time } \\
\text { and } \\
\text { Mean } \\
\text { sece }\end{array}$ & & $\begin{array}{c}\text { Number } \\
\text { of Falls in } \\
\text { Last } 6 \\
\text { Months } \\
\text { Mean (SD) }\end{array}$ \\
\hline Multiple Sclerosis & 10 & 54 & $54(11)$ & $13.9(8.4)$ & $\begin{array}{l}\text { PDDS: } 2.6(1.0), \\
\text { range } 1-4\end{array}$ & 13.88 & & $4.41(21.15)$ \\
\hline Parkinson Disease & 24 & 18 & $68(8)$ & $7.8(6.15)$ & $\begin{array}{c}\text { HY: } 2.67(0.65) \\
\text { range } 2-4\end{array}$ & 11.04 & & $3.62(8.52)$ \\
\hline Healthy Controls & 2 & 30 & $51(11)$ & NA & NA & $\begin{array}{r}6.98(0.72) ; \\
\text { age rang }\end{array}$ & & $0.06(0.24)$ \\
\hline
\end{tabular}

*Performed at "fast as possible speed while staying safe.". PDDS=Patient Determined Disease Steps, HY= Hoehn and Yahr, NA=not applicable.

four rotational forces were applied for approximately 3 seconds each in the transverse plane: clockwise and then counterclockwise at the shoulders and then pelvis. Therapists manually applied the rotational forces while subjects resisted as strongly as they could. All therapists documented LOB immediately following each of the nudges and resisted rotations using a $0-3$ rating scale that adds one category and reverses the $0-2$ scale used for the traditional nudge test (Granacher, et al., 2012). In the new scale, 0 (instead of 2 as scored on the nudge test) means stable, no loss of balance; 1-2 indicate progressively greater body movement and slower recovery of stable stance (see Fig. 1). The score of 3 includes stepping and near falls if not for therapist assist during or following the perturbation.

Six volunteer reviewers, students in a Doctor of Physical Therapy program, underwent approximately one hour of training in the perturbation protocol and use of the LOB rating scale. As part of training, all reviewers practiced using the scale to rate videotaped LOB for 2 subjects, including discussion of observations and group alignment of scoring. Reviewers then viewed assigned videos and individually scored LOB following perturbations. Reviewers determined LOB ratings for a total of 12 perturbations for each subject within each therapist: 8 nudges plus 4 resisted rotations. Reviewers did not have access to the therapists' scores when they viewed the videos. Four to five reviewers examined videos for each of the four therapists (Table 1).
Intra-class correlation coefficients $\left(\mathrm{ICC}_{2,1}\right)$ (Portney \& Watkins, 2000) using SPSS (v. 26.0) were calculated for each therapist across reviewers examining videos, and included the therapist's own scores given during the original perturbation protocol; thus, each perturbation had 5-6 ratings. In addition, percent agreement (Portney \& Watkins, 2000) across raters within each therapist was calculated by subtracting the minimum from the maximum of the 5-6 ratings for each of the perturbations across all subjects the therapist assessed (see Table 1). Agreement was determined by the proportion of perturbations that had ratings with differences of 1 or less across all raters. For example, if 2 raters rated the loss of balance as a $0(\mathrm{~min})$ and 4 rated it as a $1(\max )$, that difference was counted as agreement $(\max -\min =1)$; if the min was 0 and the max was 2, the max-min difference of 2 was not counted as agreement.

\subsection{Comparing groups and types of perturbations}

Retrospective data regarding patterns of balance loss in participants with PD (Lazaro, 2010), MS (Horn, et al., 2018), and no known neurologic disorders (healthy controls) (Crittendon, et al., 2014; Horn, et al., 2018) were collected during three separate studies according to approved protocols. The protocols for standardized perturbations were the same across all studies. All data were de-identified. 
Eligibility criteria were similar across groups. All participants must be able to follow directions, read and speak English, walk with or without an assistive device, and be free from pain or other conditions that might be exacerbated by external perturbations. Participants diagnosed with MS must self-report balance or mobility difficulties although they must be ambulatory for at least 25 feet with or without an assistive device, and physically able to endure up to three hours of testing with rest breaks. Participants with MS were excluded if they had an exacerbation within the past two months or were diagnosed with a concurrent neurological disorder such as head injury, stroke, or Parkinson's disease. Participants diagnosed with PD must be able to bear weight on both lower extremities, stand for at least 10 seconds, ambulate at least 50 feet, and have stable vital signs. Participants with PD were excluded if they had other conditions that might affect balance, spinal precautions, severe or fixed structural limitations, or severe visual or cognitive impairment. Healthy controls (HCs) had to be free from neurological diagnoses or pain that affected balance or gait.

Recruitment notices for people with MS or PD were posted in targeted newsletters and physicians' offices. Recruitment of HCs was through word of mouth and flyers posted at researchers' institutions. The number of subjects from the separate studies was 64 with MS, 42 with PD, and 32 HCs (see Table 1).

Each participant stood erect with feet together while the same therapist throughout the studies provided the standardized set of manual perturbations. LOB following each perturbation was recorded on the $0-3$ rating scale (see Fig. 1) grading severity and latency of movement at the trunk and feet.

Magnitude of LOB was calculated as the sum across all ratings; the maximum sum could be 36 for any participant if LOB were severe (rated 3) following all 12 perturbations. Prevalence of LOB was calculated as the count of perturbations with non-zero LOB ratings in any direction; the maximum count could be 12 for any participant if all perturbations resulted in LOB that was rated $1-3$. The 12 perturbations were classified by type: 6 upper and 6 lower torso perturbations (including 4 nudges and 2 rotational perturbations each), including 2 anterior and 2 posterior nudges (one of each at the upper and lower torso), 4 right and left nudges (right and left at the upper and lower torso), and 4 rotational perturbations (see Fig. 1).

Two-factor mixed design ANOVAs (SPSS v26.0) were used to compare groups, types of perturbations,

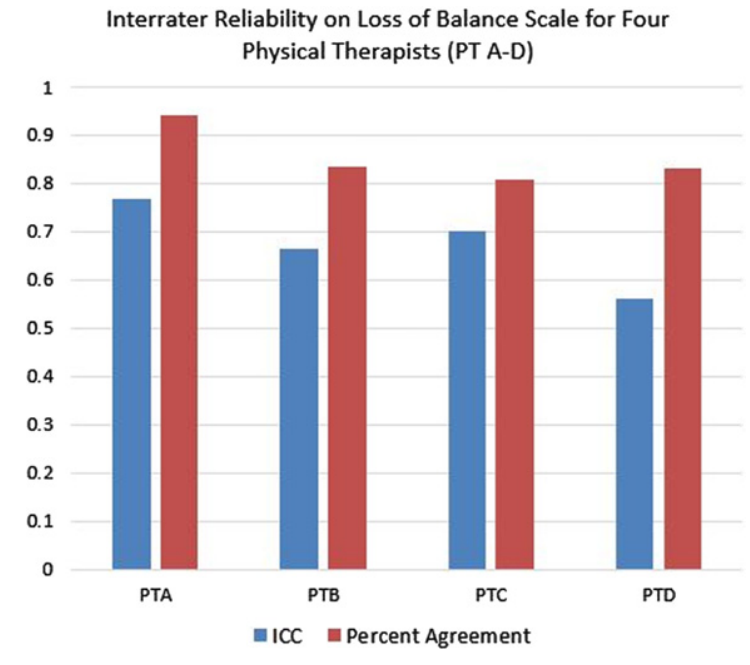

Fig. 2. Intra-class correlation coefficients (ICC) and percent agreement on Loss of Balance Scale across raters for each of four physical therapists

and interaction effects for LOB magnitude and prevalence. Magnitude and prevalence of balance loss were compared for: 1) upper vs. lower torso perturbations (including nudges and rotations); 2) anterior (A) vs. posterior (P) nudges; and 3) anterior/posterior (AP) vs. right-left (RL) nudges vs. rotational (Rots) types of perturbations. $P$-values less than 0.05 were considered significant for ANOVAs. Three planned post-hoc analyses using Dunnett T3 tests (SPSS v26.0) examined pairwise between-group significance when the main effect across the three groups was significant. Three planned post-hoc ANOVAs examined pairwise significance when the main effect of type of perturbation was significant across AP vs. RL vs. Rots. $P$-values less than 0.017 were considered significant for post-hoc analyses.

\section{Results}

\subsection{Reliability}

Reliability across five to six reviewers as assessed by $\mathrm{ICC}_{2,1}$ ranged from 0.56 to 0.77 for the four therapists (Fig. 2). Reliability as assessed by percent agreement within one point on the rating scale ranged from $81 \%$ to $94 \%$. The highest ICCs and agreement values were achieved with the most experienced therapist, who also provided perturbations for the largest number of subjects. 


\subsection{Comparing groups and types of perturbations}

Interaction, main effects, and post hoc analyses for magnitude and prevalence of LOB among the three groups and following different types of perturbations are listed in Table 2. Comparisons for magnitude and prevalence yielded similar results, with magnitude data typically showing more extreme values. Figure 3 A-C depicts results for comparisons of magnitude, only.

\subsubsection{Upper versus lower perturbations \\ (Fig. 3A: Upper v Lower)}

Interaction effects were significant among groups and types of perturbations for both magnitude and prevalence $(p<.001)$. The main effects of group and type of perturbation were both significant for magnitude and prevalence $(p<.001)$. LOB was similarly severe or frequent when perturbing participants at the upper versus the lower areas for MS and HC groups; the PD group responded to perturbations at the upper areas with greater magnitude and prevalence of balance loss compared to perturbations at the lower areas. All pairwise group differences were significant $(p<.017)$.

\subsubsection{Anterior versus posterior perturbations (Fig. 3B: $A \cup P$ )}

Interaction effects were significant among groups and types of perturbations for both magnitude and prevalence $(p<.001)$. The main effects of group and type of perturbation were both significant for magnitude and prevalence $(p<.001)$. In general, loss of balance was more severe or frequent when nudging participants backward rather than forward for MS and HC groups, with an opposite trend for the PD group. All pairwise group differences were significant except that the overall magnitude of balance loss was similar for PD and HC groups $(p>0.017)$.

\subsubsection{AP versus $R L$ versus rotational} perturbations (Fig. 3C: AP v RL v Rots)

Interaction effects were significant among groups and types of perturbations for prevalence $(p<0.05)$ but not for magnitude $(p>0.05)$. The main effects of group and type of perturbation were both significant for magnitude and prevalence $(p<.001)$. Pairwise group comparisons were all significant $(p<0.017)$.

In a post-hoc analysis of AP to RL perturbations, main effects of group and type were both significant for prevalence and magnitude. Loss of balance was greater and more frequent in response to RL perturbations compared to AP perturbations in all groups $(p<0.01)$. Pairwise group comparisons were significant except that the overall magnitude of balance loss was similar for PD and HC groups $(p>0.017)$.

In a post-hoc analysis of AP to rotational perturbations, main effects were significant for magnitude and prevalence except that the prevalence of loss of balance was not significantly different following the two types of perturbation $(p>0.017)$. Pairwise group differences were significant for MS versus PD or HC in magnitude and prevalence, and PD versus $\mathrm{HC}$ in magnitude; the prevalence of balance loss was not statistically different between the PD and HC groups $(p>0.017)$.

In a post-hoc analysis of RL to rotational perturbations, the main effect of group was significant for magnitude and prevalence. The main effect of type of perturbation was significant only for prevalence; not for magnitude $(p>0.017)$. Pairwise group differences were significant for MS versus PD or HC in magnitude and prevalence, but not for PD versus HC in either ( $p>0.017$ both magnitude and prevalence).

\section{Discussion}

\subsection{Reliability}

Ratings of observed LOB following standardized manual perturbations showed moderate to good interrater reliability and good to excellent agreement. These results support the use of this scale when assessing LOB following standardized perturbations. Clinicians and reviewers can be trained to rate LOB similarly although the most experienced clinician had the highest level of ICC and agreement across reviewers. Less experienced clinicians could consider intermittent review of the scoring rubric to increase reliability.

Visual analysis of video-taped performance has precedence in rehabilitation to establish inter-rater reliability. Davis et al. (Davis, Bridge, Miller, \& Nelson-Wong, 2011) analyzed a videotaped performance of individuals performing the hip abduction test, scored by visual analysis using a 4-point scale: their ICC values (also performed on an ordinal scale) were between 0.56 and 0.70 , very similar to the values found in this study ( 0.56 to 0.77 ). The authors concluded that the test could be reliably scored by people with different years of clinical experience $(0$ to15 years). In our study, therapist $A$ had the most 
Table 2

Two-factor Mixed Analysis of Variance and Post-hoc Comparisons of Loss of Balance in Response to Types of Perturbations by Different Groups

\begin{tabular}{|c|c|c|c|c|c|c|c|c|c|c|c|c|c|c|c|}
\hline \multicolumn{8}{|c|}{ Magnitude of Balance Loss } & \multicolumn{8}{|c|}{ Prevalence of Balance Loss } \\
\hline $\begin{array}{l}\text { Types of } \\
\text { Perturbation }\end{array}$ & $\begin{array}{l}\text { Interaction } \\
\text { and Main } \\
\text { Effects }\end{array}$ & $\begin{array}{l}\text { Post-hoc } \\
\text { Groups } \\
\text { Paired }\end{array}$ & $\mathrm{F}$ & $\begin{array}{l}\text { DF for } \\
\text { F test: } \\
\text { test/error }\end{array}$ & $\begin{array}{c}\text { Mean } \\
\text { Pair-wise } \\
\text { Difference }\end{array}$ & $\begin{array}{c}95 \% \\
\text { Confidence } \\
\text { Interval }\end{array}$ & $\mathrm{P}$ & $\begin{array}{l}\text { Types of } \\
\text { Perturbation }\end{array}$ & $\begin{array}{l}\text { Interaction } \\
\text { and Main } \\
\text { Effects }\end{array}$ & $\begin{array}{l}\text { Post-hoc } \\
\text { Groups } \\
\text { Paired }\end{array}$ & $\mathrm{F}$ & $\begin{array}{l}\text { DF for } \\
\text { F test: } \\
\text { test/error }\end{array}$ & $\begin{array}{c}\text { Mean } \\
\text { Pair-wise } \\
\text { Difference }\end{array}$ & $\begin{array}{c}95 \% \\
\text { Confidence } \\
\text { Interval }\end{array}$ & $P$ \\
\hline \multirow[t]{6}{*}{ Upper v Lower } & Gr x Type & & 26.28 & $2 / 135$ & & & $<0.001$ & Upper v Lower & Gr x Type & & 20.64 & $2 / 135$ & & & $<0.001$ \\
\hline & Type & & 14.23 & $1 / 135$ & & & $<0.001$ & & Type & & 39.07 & $1 / 135$ & & & $<0.001$ \\
\hline & Group & & 108.26 & $2 / 135$ & & & $<0.001$ & & Group & & 66.15 & $2 / 135$ & & & $<0.001$ \\
\hline & & MS v PD & & & 5.71 & $4.67,6.75$ & $<0.001$ & & & MS v PD & & & 2.29 & $1.75,2.83$ & $<0.001$ \\
\hline & & $\mathrm{MS}$ v $\mathrm{HC}$ & & & 4.53 & $3.56,5.50$ & $<0.001$ & & & MS v HC & & & 1.50 & $0.98,2.02$ & $<0.001$ \\
\hline & & $\mathrm{PD}$ v $\mathrm{HC}$ & & & -1.18 & $-2.17,-0.19$ & 0.014 & & & PD v HC & & & -0.79 & $-1.42,-0.15$ & 0.01 \\
\hline \multirow[t]{6}{*}{$A \vee P$} & Gr x Type & & 24.81 & $2 / 135$ & & & $<0.001$ & $A \vee P$ & Gr x Type & & 19.14 & $2 / 135$ & & & $<0.001$ \\
\hline & Type & & 40.52 & $1 / 135$ & & & $<0.001$ & & Type & & 32.70 & $1 / 135$ & & & $<0.001$ \\
\hline & Group & & 42.93 & $2 / 135$ & & & $<0.001$ & & Group & & 47.67 & $2 / 135$ & & & $<0.001$ \\
\hline & & MS v PD & & & 1.85 & $1.34,2.35$ & $<0.001$ & & & MS v PD & & & 0.96 & $0.73,1.18$ & $<0.001$ \\
\hline & & $\mathrm{MS} \vee \mathrm{HC}$ & & & 1.45 & $0.92,1.99$ & $<0.001$ & & & $\mathrm{MS} v \mathrm{HC}$ & & & 0.57 & $0.28,0.86$ & $<0.001$ \\
\hline & & $\mathrm{PD}$ v HC & & & -0.39 & $-0.88,0.09$ & 0.140 & & & PD v HC & & & -0.39 & $-0.69,-0.09$ & 0.008 \\
\hline \multirow{6}{*}{ AP v RL v Rots } & Gr x Type & & $0.89^{*}$ & $4 / 270$ & & & 0.473 & AP v RL v Rots & Gr x Type & & $2.92^{*}$ & $4 / 270$ & & & 0.022 \\
\hline & Type & & $7.73^{*}$ & $2 / 134$ & & & 0.001 & & Type & & $21.28^{*}$ & $2 / 134$ & & & $<0.001$ \\
\hline & Group & & 108.26 & $2 / 135$ & & & $<0.001$ & & Group & & 66.15 & $2 / 135$ & & & $<0.001$ \\
\hline & & MS v PD & & & 3.81 & $3.11,4.50$ & $<0.001$ & & & MS v PD & & & 1.53 & $1.17,1.88$ & $<0.001$ \\
\hline & & MS v HC & & & 3.02 & $2.37,3.67$ & $<0.001$ & & & MS v HC & & & 1.00 & $0.65,1.35$ & $<0.001$ \\
\hline & & $\mathrm{PD} \vee \mathrm{HC}$ & & & -0.79 & $-1.44,-0.13$ & 0.014 & & & PD v HC & & & -0.53 & $-0.95,-0.10$ & 0.01 \\
\hline \multicolumn{8}{|c|}{ Post-hoc Types Paired } & Post-hoc Types P & aired & & & & & & \\
\hline \multirow[t]{5}{*}{$\overline{A P \vee R L}$} & Type & & 15.30 & $1 / 135$ & & & $<0.001$ & $A P \vee R L$ & Type & & 39.12 & $1 / 135$ & & & $<0.001$ \\
\hline & Group & & 78.29 & $2 / 135$ & & & $<0.001$ & & Group & & 70.21 & $2 / 135$ & & & $<0.001$ \\
\hline & & MS v PD & & & 3.87 & $3.06,4.68$ & $<.001$ & & & MS v PD & & & 1.79 & $1.42,2.16$ & $<.001$ \\
\hline & & MS v HC & & & 3.23 & $2.39,4.08$ & $<0.001$ & & & MS v HC & & & 1.09 & $0.64,1.54$ & $<0.001$ \\
\hline & & $\mathrm{PD} \vee \mathrm{HC}$ & & & -0.63 & $-1.45,0.18$ & 0.172 & & & $\mathrm{PD}$ v $\mathrm{HC}$ & & & -0.70 & $-1.2,-0.19$ & 0.004 \\
\hline \multirow[t]{5}{*}{ AP v Rots } & Type & & 6.33 & $1 / 135$ & & & 0.013 & AP v Rots & Type & & 3.06 & $1 / 135$ & & & 0.082 \\
\hline & Group & & 82.75 & $2 / 135$ & & & $<0.001$ & & Group & & 48.08 & $2 / 135$ & & & $<0.001$ \\
\hline & & MS v PD & & & 3.69 & $2.93,4.45$ & $<0.001$ & & & MS v PD & & & 1.45 & $1.05,1.86$ & $<0.001$ \\
\hline & & MS v HC & & & 2.75 & $2.05,3.45$ & $<0.001$ & & & MS v HC & & & 0.98 & $0.62,1.34$ & $<0.001$ \\
\hline & & $\mathrm{PD} \vee \mathrm{HC}$ & & & -0.94 & $-1.66,-0.22$ & 0.006 & & & $\mathrm{PD}$ v HC & & & -0.48 & $-0.91,-0.04$ & 0.029 \\
\hline \multirow[t]{5}{*}{ RL v Rots } & Type & & 0.42 & $1 / 135$ & & & 0.520 & RL v Rots & Type & & 13.91 & $1 / 135$ & & & $<0.001$ \\
\hline & Group & & 88.48 & $2 / 135$ & & & $<0.001$ & & Group & & 40.80 & $2 / 135$ & & & $<0.001$ \\
\hline & & MS v PD & & & 3.86 & $3.09,4.63$ & $<0.001$ & & & MS v PD & & & 1.33 & $0.92,1.74$ & $<0.001$ \\
\hline & & MS v HC & & & 3.08 & $2.34,3.82$ & $<0.001$ & & & MS v HC & & & 0.93 & $0.55,1.31$ & $<0.001$ \\
\hline & & $\mathrm{PD}$ v HC & & & -0.79 & $-1.52,-0.05$ & 0.033 & & & PD v HC & & & -0.40 & $-0.88,0.07$ & 0.115 \\
\hline
\end{tabular}

$\mathrm{DF}=$ degrees of freedom, $A=$ anterior, $P=$ posterior, $\mathrm{Gr}=$ group, $\mathrm{MS}=$ multiple sclerosis, $\mathrm{PD}=$ Parkinson's disease, $\mathrm{HC}=$ healthy controls, $v=$ versus, $\mathrm{AP}=$ anterior-posterior, $\mathrm{RL}=$ right-left Rots $=$ rotations. ${ }^{*}$ Mauchly's test of sphericity indicated that sphericity could not be assumed; F statistic drawn from multivariate analysis, Pillai's Trace (SPSS v26). 

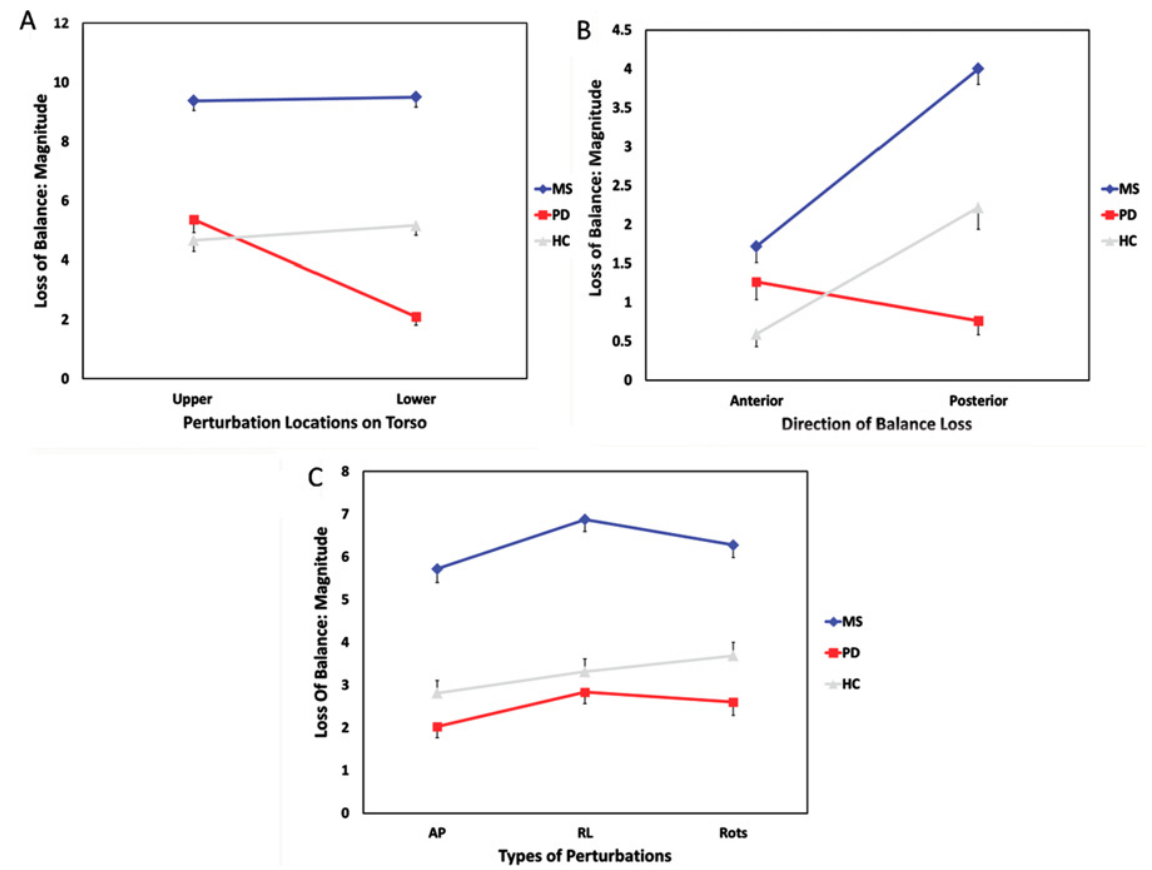

Fig. 3. A-C. Mean (SE) magnitude of balance loss with perturbations applied: A) at upper/lower torso; B) in anterior/posterior directions; $\mathrm{C}$ ) in different directions at both upper and lower torso: $\mathrm{AP}=$ forward/backward, $\mathrm{RL}=$ left/right, Rots = resisted rotations clockwise/counterclockwise. $\mathrm{MS}=$ multiple sclerosis, $\mathrm{PD}=$ Parkinson's disease, $\mathrm{HC}=$ healthy controls

experience using the perturbation testing and showed the highest agreement with the reviewers of the videotapes. The videotape reviewers were all students in a Doctor of Physical Therapy program who had at least 22 weeks of full-time supervised clinical experience prior to their one-hour training in rating LOB. Despite their limited experience in evaluating balance compared to licensed clinicians, strong agreement was still observed between the student reviewers and therapist A, suggesting that clinicians with more experience evaluating balance might require only minimal training to rate LOB similarly. Other differences between therapist $\mathrm{A}$ and the other therapists included: more perturbations performed by therapist A who assessed 10 subjects instead of 6-7 (see Table 1); four reviewers assigned to therapist A compared with five reviewers assigned to therapists B-D. The same experienced therapist (A) from the reliability portion of this study provided the LOB ratings for all three groups in the comparison portion of this study.

\subsection{Comparing groups and types of perturbations}

The 4-point LOB rating scale shows differences among MS, PD, and $\mathrm{HC}$ groups following manual perturbation of different types: upper versus lower torso, anterior versus posterior nudges, and AP versus $\mathrm{RL}$ versus rotational perturbations. The magnitude and prevalence of balance loss was consistently different across groups, with MS showing the greatest balance loss regardless of perturbation type. One reason for greater balance loss in MS than in PD could be that MS more frequently affects somatosensation (Zackowski, Wang, McGready, Calabresi, \& Newsome, 2015) and sensory processing-important aspects of reactive balance. Cattaneo et al. (Cattaneo, et al., 2016) supported this hypothesis, showing greater instability in MS than in PD or stroke when sensory systems were challenged. While our study did not challenge participants with eyes closed or foam standing conditions, perturbations also challenge stability; the PD group may have been able to utilize proprioceptive information to maintain balance more effectively than the MS group. In our samples, the PD group averaged 14 years older, which could have influenced sensory processing (Matsuda, Verrall, Finlayson, Molton, \& Jensen, 2015); however, potential sensory losses with age did not surpass the sensory deficits associated with MS. Our MS group may have been more impaired because the inclusion criteria specified self-reported mobility 
problems only in the MS group, but both our MS and PD groups were ambulatory and had mild to moderate levels of disability, with scores on the Patient Determined Disease Steps Scale and the Hoehn and Yahr scale of 1-4 and 2-4, respectively.

As expected, the MS group showed more LOB than the HC group regardless of the type of perturbation. Previous research reports that even people with mild disease severity in MS have greater balance dysfunction compared to healthy controls (Fjeldstad, Pardo, Bemben, \& Bemben, 2011). Because impaired reactive control with MS can cause LOB after perturbations, reducing fall risk is a critical early priority in rehabilitation.

In our study, the magnitude and prevalence of balance loss during standing perturbations was generally less in the PD compared to the HC group. Researchers suggest that PD may show less postural sway than HCs because of greater antagonist muscle activity (Horak, Nutt, \& Nashner, 1992) or stiffness in the axial muscles (Cattaneo, et al., 2016). However, a systematic review of postural sway using posturography revealed mixed findings: PD sway was less than, equal to, or more than the sway of healthy individuals (Kamieniarz et al., 2018). Thus, the HCs' lower LOB with perturbations in our study have precedents, and do not necessarily indicate that HCs have worse functional balance than people with PD. Specifically, falls were more prevalent in both MS and PD groups than in the HC group. Further, the average (SD) TUG values (see Table 1) for the MS and PD groups are similar at 13.88 (8.7) and 11.04 (6.47), respectively, close to the 11.5 second cut-off for increased fall risk for PD (Nocera et al., 2013). The TUG values in a sub-sample of HCs were 6.98 (0.72) seconds.

Patterns of LOB differed across types of perturbations. All groups had greater LOB with RL compared to AP perturbations. This may reflect the narrower base of support in the RL direction when people stand with feet together as they did in our protocol. Alternatively, the RL direction may be inherently less stable. Difference in RL sway compared to HCs has been noted previously in people with MS (Crittendon, et al., 2014) and PD (Błaszczyk, Orawiec, Duda-Kłodowska, \& Opala, 2007; Rossi-Izquierdo et al., 2016). Posturography via body worn gyrometers showed greater trunk sway in the medial-lateral direction when people with PD performed balancechallenging activities, although the tasks did not specifically require reactive responses to external perturbations (Rossi-Izquierdo, et al., 2016).
Two groups, HC and MS, showed similar LOB with respect to upper versus lower perturbations and greater LOB for posterior versus anterior perturbations. In contrast, the PD group showed greater LOB following upper versus lower perturbations and tended to have greater LOB for anterior perturbations. One explanation for the PD difference might be in the initial posture: persons with PD may have more trunk, hip, and knee flexion and hold the center of mass a little more anterior over the base of support when challenged to minimize posterior LOB (Błaszczyk, et al., 2007; Cattaneo, et al., 2016). Center of pressure was not recorded for this group so testing this hypothesis requires future investigation.

The LOB pattern in Fig. 3C looks similar for PD and MS: the magnitude of balance loss appears greatest for RL perturbation followed by rotations and then AP perturbations. In contrast, the $\mathrm{HC}$ group had a different pattern from either of the other groups, tending to have greater LOB with rotational perturbations. Future studies could explore this finding further.

\subsection{Study limitations}

Rating LOB following manual application of perturbations is a potential limitation of this study. However, the same experienced clinician performed all perturbations in the comparison portion of this study, and has shown good consistency in force production when tested via dynamometer (Crittendon, et al., 2014). Further, the reliability portion of the current study showed that LOB ratings are reproducible.

For our purpose, rating LOB following manual perturbations had advantages. Our protocol required no expensive equipment and yet offered a reproducible way to identify patterns of directional balance loss. Neither the nudge test at the sternum or thoracic spine (Granacher, et al., 2012; Tinetti, 1986), nor most computerized posturography applies RL perturbations, common directions of instability in MS and PD confirmed in our study. Perturbations from a force platform do not apply nudges at shoulder or pelvic height, common areas for bumps and shoves in everyday life. Sibley et al.(Sibley, Straus, Inness, Salbach, \& Jaglal, 2013) report that less than half of physical therapists assess reaction to external perturbation when testing balance impairment. Perhaps routine clinical use of quick manual perturbations in various directions will reveal a patient's reactive balance vulnerabilities to guide targeted balance challenges.

LOB was rated based on the first response to multidirectional perturbations, not an average response to 
the same perturbation (Tinetti, 1986). Our intent was to mimic unexpected perturbations that people might encounter in everyday life (Allum, Tang, Carpenter, Oude Nijhuis, \& Bloem, 2011). Although our 0-3 rating scale separated observed LOB into one more category than the traditional 0-2 nudge test (Granacher, et al., 2012), some clinicians wanting even more granularity argue that additional splitting of categories is needed to differentiate between stepping and falling. However, our 0-3 scale facilitated adequate reliability and our standardized perturbations did not normally result in falls in HCs. Based on information from the therapists in our reliability study, only $4 \%$ of the 356 perturbations viewed in these $\mathrm{HCs}$ resulted in LOB considered a near-fall.

\subsection{Clinical implications}

The current study suggests that perturbations applied to different areas of the torso (upper versus lower) and in different directions elicit different reactive responses, and that the response patterns differ in various populations. Understanding the directional deficits in reactive postural control can lead to more focused, individualized balance interventions. Specifically, if people with PD have greater LOB to perturbations applied to the upper versus lower torso or in the anterior versus posterior directions, then targeting reactive control with challenges at the upper torso and anteriorly may be most effective for reducing falls. In contrast, people with MS likely need balance challenges at both the upper and lower torso, and more practice with posteriorly directed perturbations. Both groups need practice with RL perturbations to promote better reactive control with less LOB. Clinicians must routinely test reactive balance control with perturbations to determine the most effective targeted interventions.

Perturbation-based training can improve balance and perhaps reduce falls in people with neurologic dysfunction (Mansfield, Wong, Bryce, Knorr, \& Patterson, 2015; Tajali et al., 2018). Studies indicate that challenging balance using perturbations can result in improved gait and postural stability for older adults (Mansfield, Peters, Liu, \& Maki, 2010; Mansfield, et al., 2015; Rieger, Papegaaij, Pijnappels, Steenbrink, \& van Dieën, 2020), people with PD (Gaßner et al., 2019; Mansfield, et al., 2015), and people with MS (Van Liew, Dibble, Hunt, Foreman, \& Peterson, 2019). Perturbation-based training to date has utilized mechanical perturbations that may not be available to many clinicians or in clinical settings. Further, perturbations have been uniform within studies rather than specific to the direction and location of LOB vulnerability in each individual. Assessing individuals' directional LOB using a clinically accessible and reliable rating scale may allow more targeted perturbation-based training to optimize benefits.

\section{Conclusion}

A clinically accessible and reliable rating scale revealed differences in patterns of LOB following manual perturbations in people with MS, PD, and HC. Clinicians may help reduce fall risk for people with MS or PD more effectively if they understand and address the differences in LOB following perturbations in these populations. Further, our results suggest that perturbation-based training may benefit from specific application of perturbations according to the direction and location of LOB.

\section{Acknowledgments}

The authors would like to gratefully acknowledge the participants in this study. This study was partially funded by NIH grant \#1R15HD066397-01 and SMU AVP Faculty Incentive Award.

\section{Conflict of interest}

None to report.

\section{References}

Allum, J. H., Tang, K. S., Carpenter, M. G., Oude Nijhuis, L. B., \& Bloem, B. R. (2011). Review of first trial responses in balance control: influence of vestibular loss and Parkinson's disease. Hum Mov Sci, 30(2), 279-295. doi: 10.1016/j.humov. 2010.11.009

Błaszczyk, J. W., Orawiec, R., Duda-Kłodowska, D., \& Opala, G. (2007). Assessment of postural instability in patients with Parkinson's disease. Exper Brain Res, 183(1), 107-114.

Cameron, M. H., \& Lord, S. R. (2010). Postural control in multiple sclerosis: implications for fall prevention. Curr Neurol Neurosci Rep, 10(5), 407-412.

Carpenter, M. G., Allum, J. H. J., Honegger, F., Adkin, A. L., \& Bloem, B. R. (2004). Postural abnormalities to multidirectional stance perturbations in Parkinson's disease. J Neurol Neurosurg Psychiatry, 75, 1245-1254. doi: 10.1136/jnnp.2003. 021147 
Cattaneo, D., Carpinella, I., Aprile, I., Prosperini, L., Montesano, A., \& Jonsdottir, J. (2016). Comparison of upright balance in stroke, Parkinson and multiple sclerosis. Acta Neurologica Scandinavica, 133, 346-354.

Crittendon, A., O’Neill, D., Widener, G. L., \& Allen, D. D. (2014). Standing data disproves biomechanical mechanism for balance-based torso-weighting. Archives of Physical Medicine \& Rehabilitation, 95, 43-49.

Davis, A. M., Bridge, P., Miller, J., \& Nelson-Wong, E. (2011). Interrater and intrarater reliability of the active hip abduction test. J Orthop Sports Phys Ther, 41(12), 953-960. doi: 10.2519/jospt.2011.3684

Dietz, V., Berger, W., \& Horstmann, G. A. (1988). Posture in Parkinson's disease: Impairment of reflexes and programming. Annals of Neurology, 24, 660-669.

Fjeldstad, C., Pardo, G., Bemben, D., \& Bemben, M. (2011). Decreased postural balance in multiple sclerosis patients with low disability. Int $J$ Rehabil Res, 34(1), 53-58. doi: 10.1097/MRR.0b013e32833d6ccb

Gaßner, H., Steib, S., Klamroth, S., Pasluosta, C. F., Adler, W., Eskofier, B. M., Pfeifer, K., Winkler, J., Klucken, J. (2019). Perturbation treadmill training improves clinical characteristics of gait and balance in Parkinson's disease. J Parkinsons Dis, 9(2), 413-426. doi: 10.3233/JPD-181534

Gibson-Horn, C. (2008). Balance-based torso-weighting in a patient with ataxia and multiple sclerosis: a case report. Journal of Neurologic Physical Therapy, 32, 139-146.

Gorgas, A.-M., Widener, G. L., Gibson-Horn, C., \& Allen, D. D. (2015). Gait changes with balance-based torso-weighting in people with multiple sclerosis. Physiotherapy Research International, 20(1), 45-53.

Granacher, U., Muehlbauer, T., \& Gruber, M. (2012). A qualitative review of balance and strength performance in healthy older adults: impact for testing and training. Journal of Aging Research, 2012(Article ID 708905), 16 pages.

Horak, F. B., Nutt, J. G., \& Nashner, L. M. (1992). Postural inflexibility in parkinsonian subjects. J Neurol Sci., 111(1), 46-58. doi: 10.1016/0022-510x(92)90111-w

Horn, K. K., Allen, D. D., Gibson-Horn, C., \& Widener, G. L. (2018). Effects of torso-weighting on standing balance and falls during the Sensory Organization Test in people with multiple sclerosis. International Journal of MS Care, 20, 68-75. doi: 10.7224/1537-2073.2015-090

Kalilani, L., Asgharnejad, M., Palokangas, T., \& Durgin, T. (2016). Comparing the incidence of falls/fractures in Parkinson's disease in patients in the US population. PLoS One, 11(9), e0161689. doi: 10.1371/journal.pone.0161689

Kamieniarz, A., Michalska, J., Brachman, A., Pawłowski, M., Słomka, K. J., \& Juras, G. (2018). A posturographic procedure assessing balance disorders in Parkinson's disease: a systematic review Clin Interv Aging, 13, 2301-2316. doi: 10.2147/ CIA.S180894

Lazaro, R. (2010). The Immediate Effects of Torso-Weighting on Balance and Mobility Measures of People with Parkinson's Disease doctoral, Touro University, Cypress, CA.

Mansfield, A., Peters, A. L., Liu, B. A., \& Maki, B. E. (2010). Effect of a perturbation-based balance training program on compensatory stepping and grasping reactions in older adults: a randomized controlled trial. Phys Ther, 90(4), 476-491. doi: 10.2522/ptj.20090070

Mansfield, A., Wong, J. S., Bryce, J., Knorr, S., \& Patterson, K. K. (2015). Does perturbation-based balance training prevent falls? Systematic review and meta-analysis of preliminary randomized controlled trials. Phys Ther, 95, 700-709. doi: 10.2522/ptj.20140090

Matsuda, P. N., Verrall, A. M., Finlayson, M., Molton, I. R., \& Jensen, M. P. (2015). Falls among adults aging with disability. Archives of Physical Medicine \& Rehabilitation, 96, 464-471.

Nilsagard, Y., Gunn, H., Freeman, J. A., Lord, S., Mazumder, R., \& Cameron, M. H. (2015). Falls in people with MS-an individual data meta-analysis from studies from Australia, Sweden, United Kingdom and the United States. Multiple Sclerosis Journal, 21, 92-100.

Nocera, J. R., Stegemöller, E. L., Malaty, I. A., Okun, M. S., Marsiske, M., Hass, C. J., \& National Parkinson Foundation Quality Improvement Initiative Investigators. (2013). Using the Timed Up \& Go test in a clinical setting to predict falling in Parkinson's disease. Arch Phys Med Rehabil, 94(7), 1300-1305. doi: 10.1016/j.apmr.2013.02.020

Peterson, D. S., Huisinga, J. M., Spain, R. I., \& Horak, F. B. (2016). Characterization of compensatory stepping in people with multiple sclerosis. Archives of Physical Medicine \& Rehabilitation, 97, 513-521.

Pickering, R. M., Grimbergen, Y. A. M., Rigney, U., Ashburn, A., Mazibrada, G., Wood, B., Gray, P., Kerr, G., Bloem, B. R. (2007). A meta-analysis of six prospective studies of falling in Parkinson's disease. Mov. Disord., 22, 1892-1900.

Portney, L. G., \& Watkins, M. P. (2000). Foundations of Clinical Research: Applications to Practice (2nd ed.). Upper Saddle River, NJ: Prentice Hall Health.

Rieger, M. M., Papegaaij, S., Pijnappels, M., Steenbrink, F., \& van Dieën, J. H. (2020). Transfer and retention effects of gait training with anterior-posterior perturbations to postural responses after medio-lateral gait perturbations in older adults. Clin Biomech (Bristol, Avon), 75, 104988. doi: 10.1016/j.clinbiomech.2020.104988

Rossi-Izquierdo, M., Soto-Varela, A., Ernst, A., Rubio-Rodríguez, J. P., Santos-Pérez, S., Sesar, Á., Alberte-Woodward, M., Guijarro-Del Amo, M., San Román-Rodríguez, E., FaraldoGarcía, A., Zubizarreta-Gutiérrez, A., Basta, D. (2016). What could posturography tell us about balance problems in Parkinson's disease? Otol Neurotol, 37(9), e326-e331. doi: 10.1097/MAO.0000000000001120

Shirley Ryan AbilityLab. (2012, August 06, 2012). Push and Release Test. Rehabilitation Measures Database Retrieved February 6, 2021, from sralab.org/rehabilitation-measures/ push-and-release-test

Sibley, K. M., Straus, S. E., Inness, E. L., Salbach, N. M., \& Jaglal, S. B. (2013). Clinical balance assessment: perceptions of commonly-used standardized measures and current practices among physiotherapists in Ontario, Canada. Implementation Sci, 8(33). doi: 10.1186/1748-5908-8-33

Tajali, S., Rouhani, M., Mehravar, M., Negahban, H., Sadati, E., \& Oskouei, A. E. (2018). Effects of external perturbations on anticipatory and compensatory postural adjustments in patients with multiple sclerosis and a fall history. Int J MS Care, 20(4), 164-172. doi: 10.7224/1537-2073.2016-098

Tinetti, M. E. (1986). Performance-oriented assessment of mobility problems in elderly patients. J Am Geriatr Soc, 34(2), 119-126.

Van Liew, C., Dibble, L. E., Hunt, G. R., Foreman, K. B., \& Peterson, D. S. (2019). Protective stepping in multiple sclerosis: Impacts of a single session of in-place perturbation practice. 
Mult Scler Relat Disord, 30, 17-24. doi: 10.1016/j.msard. 2019.01.054

Vincenzo, J. L., Gibson-Horn, C., \& Gray, M. (2017). Short-term effect of BalanceWear therapy on mobility in older adults with mobility limitations. Journal of Geriatric Physical Therapy, 40, 175-182. doi: 10.1519/JPT.0000000000000094

Widener, G. L., Allen, D. D., \& Gibson-Horn, C. (2009a). Balancebased torso-weighting may enhance balance in persons with multiple sclerosis: preliminary evidence. Archives of Physical Medicine and Rehabilitation, 90, 602-609.

Widener, G. L., Allen, D. D., \& Gibson-Horn, C. (2009b). Randomized clinical trial of balance-based torso weighting for improving upright mobility in people with multiple sclerosis Neurorehabilitation and Neural Repair, 23(8), 784-791.

Widener, G. L., Conley, N., Whiteford, S., Gee, J., Harrell, A., Gibson-Horn, C., Block, V., Allen, D. D. (2020). Changes in standing stability with balance-based torso-weighting in people with cerebellar ataxia: a pilot study. Physiotherapy Research International, 25(1), e1814. doi: 10.1002/pri.1814

Zackowski, K. M., Wang, J. I., McGready, J., Calabresi, P. A., \& Newsome, S. D. (2015). Quantitative sensory and motor measures detect change over time and correlate with walking speed in individuals with multiple sclerosis. Mult Scler Relat Disord, 4(1), 67-74. doi: 10.1016/j.msard.2014.11.001 